\title{
Ichthyological ethnoknowledge of the "piabeiros" from the Amazon region, Brazil
}

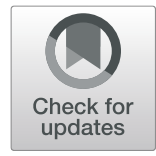

\author{
Daniel da Silva Ladislau' ${ }^{1}$, Maiko Willas Soares Ribeiro ${ }^{2} \mathbb{D}$, Philip Dalbert da Silva Castro ${ }^{3} \mathbb{D}$, \\ Jackson Pantoja-Lima ${ }^{4}$ (D) Paulo Henrique Rocha Aride $^{5}$ (I) and Adriano Teixeira de Oliveira ${ }^{5^{*}}$ (D)
}

\begin{abstract}
Background: The capture of ornamental fish is one of the main economic activities of riverine families in the Amazon. However, studies regarding the local ecological knowledge of workers in this activity are still incipient. In view of this, we have studied and explored the local ecological knowledge of artisanal fishers who specialize in the capture of fish for the aquarium trade in the middle part of the Negro River basin and investigated issues related to the ecological aspects of the fish species that are targeted by this trade in the region.

Methods: Therefore, we conducted semi-structured interviews and applied questionnaires to artisanal fishers of ornamental fish ( $N=89)$, from the municipality of Barcelos, from January to April 2016.

Results: In total, 41 popular names were cited, which correspond to four ethnocategories and 10 families. The main species were Paracheirodon axelrodi (12.5\%), Hemigrammus bleheri (8.3\%), Ancistrus dolichopterus (6.4\%),

Symphysodon discus (5.3\%), and Potamotrygon motoro (3.8\%). According to the fishers, the species of fish known in the region as "piabas" have a preference for living in clusters (28.9\%) and carry out migratory movements (26.1\%). The diet of local fish species reported by fisheries is diverse, though mainly based on periphyton (42.2\%), and the reproductive cycle directly influenced by the period of flooding of rivers in the region (37.6\%)

Conclusion: Our study revealed that the fishers possess information on the ecological aspects of local ornamental fish species, many of which are consistent with scientific literature. The information presented may assist in the decision-making process for the management of local fishery resources and contribute to the resumption of growth and sustainability in the capture of ornamental fish.
\end{abstract}

Keywords: Ornamental fish, Artisanal fishers, Ethnoichthyology, Amazon

\section{Introduction}

Ethnoichthyology is an ethnoscience (a branch of ethnozoology) that aims to study the knowledge, use, and meaning of ichthyofauna for different human populations, in regard to behavioral and cognitive aspects [1]. In Brazil, ethnoichthyology is a field that has only recently started to be explored when compared to other countries; however, this and other ethnosciences have

\footnotetext{
* Correspondence: adriano.oliveira@ifam.edu.br

${ }^{5}$ Instituto Federal de Educação, Ciência e Tecnologia do Amazonas, Manaus, AM 69020-120, Brazil

Full list of author information is available at the end of the article
}

grown significantly in the country since the last decade, as demonstrated by the increase in publications and studies, which contribute to the consolidation of ethnobiology in Latin America [2-5].

Ethnoichthyological studies have contributed via new information on the biological and ecological aspects of the target species of tropical fisheries. This has been beneficial since data on fishing in coastal and continental areas are often not reliable, and the lack of information hinders the process of decision-making in management plans [6]. Thus, the ecological knowledge of fishers from various regions of the country has been used in fisheries

\section{BMC}

(c) The Author(s). 2021 Open Access This article is licensed under a Creative Commons Attribution 4.0 International License, which permits use, sharing, adaptation, distribution and reproduction in any medium or format, as long as you give appropriate credit to the original author(s) and the source, provide a link to the Creative Commons licence, and indicate if changes were made. The images or other third party material in this article are included in the article's Creative Commons licence, unless indicated otherwise in a credit line to the material. If material is not included in the article's Creative Commons licence and your intended use is not permitted by statutory regulation or exceeds the permitted use, you will need to obtain permission directly from the copyright holder. To view a copy of this licence, visit http://creativecommons.org/licenses/by/4.0/ The Creative Commons Public Domain Dedication waiver (http://creativecommons.org/publicdomain/zero/1.0/) applies to the data made available in this article, unless otherwise stated in a credit line to the data. 
management, due to the fact that these actors have a detailed knowledge of the ecological, behavioral and classificatory aspects of fish [5-7].

In the Amazon, the capture of ornamental fish is responsible for the livelihood of a number of fishing communities, and occurs in the main tributaries of the Xingu [8, 9], Tapajós [10], Solimões [11], Purus [12] and Negro Rivers [13-20]. In the middle of the Negro River region, this activity is artisanal, selective and practiced by local fishers who are popularly known in the region as "piabeiros". These fishers, through years of experience, have acquired a deep knowledge about local natural resources $[15,19]$.

In the Amazon Rainforest there are only two seasons: wet and dry, and the start of the dry season is when most of the fishing for piaba takes place [21]. For the fish that are captured, it is somewhat similar to a rescue operation; for example, a cardinal tetra (Paracheirodon axeroldi (Schultz, 1956)), one of the most popular piaba species, would be lucky to survive a year in the wild [21]. In a home aquarium, a cardinal tetra might live to two, three, or more years. In this sense, it may be considered to be the world's most benign fishery [21]. Chao et al. [22] affirm that the trade in ornamental fish in the Amazon is fundamental to the maintenance of the forest. The authors created the slogan "buy a fish, save a tree," which implies that the ornamental fish trade provides income for the riverine population and thus avoids the need to cut down trees as a means of income.
Despite the recognition of the importance of the capture of ornamental fish in the Amazon region, there are still few studies that focus on local ecological knowledge of artisanal fishers in the region [9, 12, 17]. In addition, the Negro River has a rich and diversified ichthyofauna with approximately 1,165 species. Many of these species are endemic to this basin and have not yet been cataloged or described, and some are captured and traded for the international aquarium market [23]. The traditional knowledge of riverine communities regarding the ecological aspects of species is often ignored by fisheries resource managers, thus causing relevant information to be lost.

In view of this, we have studied and explored the local ecological knowledge of artisanal fishers who specialize in the capture of fish for the aquarium trade in the middle part of the Negro River basin, and investigated issues related to the ecological aspects of the fish species that are targeted by this trade in the region.

\section{Materials and methods}

\section{Study area}

The present study was carried out in the urban and riverine areas of the municipality of Barcelos (Fig. 1). Barcelos was the first capital of the state of Amazonas from 1758 until 1808. It is located on the right bank of the Negro River, $496 \mathrm{~km}$ from the capital Manaus by river and, in regard to territorial extension, it is considered the largest municipality in the Amazonas state with

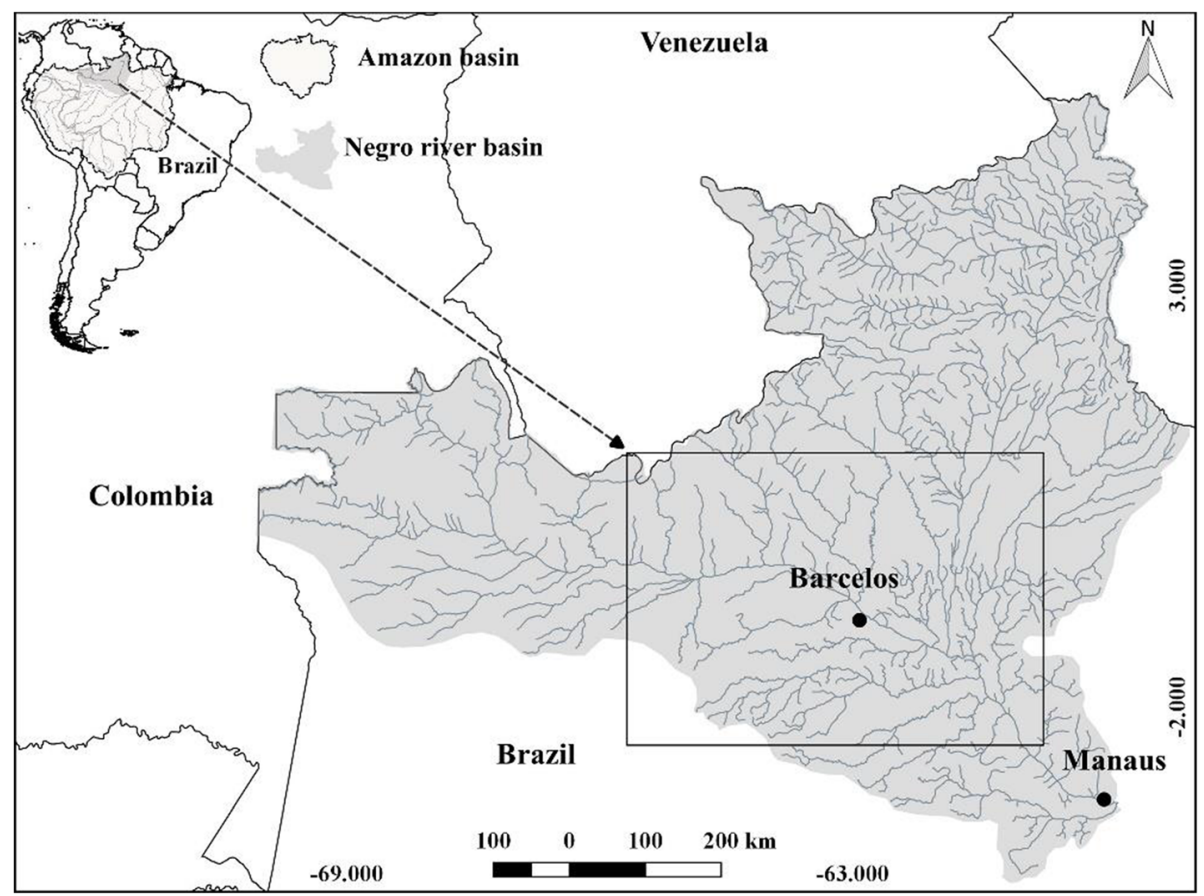

Fig. 1 Region of the municipality of Barcelos located in the Negro River basin, Amazonas state, Brazil 
$112,450,769 \mathrm{~km}^{2}$, and has a population of 25,589 thousand inhabitants [24].

The exploration of ornamental fish in Barcelos started with Herbet Axelrod in 1955, when he visited the region looking for discus fish, probably the Symphysodon discus (Heckel, 1840), but ended up discovering the cardinal tetra $P$. axeroldi [22]. Within a year, the export business was flourishing, and a fishing operation was set up in Barcelos, which employed 50 workers [22]. The connection with the Negro River and the cardinal tetra continues to this day though, and ensures the preservation of the piaba and its habitat, as well the well-being of the local fishers [22].

\section{Interviews}

All stages of this study received authorization and followed the protocols involving human beings established by the Research Ethics Committee (CEP) and was registered on "Plataforma Brasil" ( $\mathrm{N}^{\mathrm{o}} .53847316 .6 .0000 .5015$ and 2.238.505). Semi-structured interviews were conducted with the application of questionnaires to artisanal ornamental fishers $(N=89)$ in the urban and riverine areas of the municipality of Barcelos, in the period from January to April, 2016 (Table 1).

The participants in this research were randomly selected in the city of Barcelos and in the riverine communities; however, in this study we considered only the information from artisanal fishers who identified themselves as "piabeiros" (Fig. 2). The interviewees were asked questions related to the ecological aspects (behavior, diet, reproduction) of the species of fish targeted in the ornamental fish trade in the region, such as, a) Which fish species do you fish for? (a) Do you catch fish for the local aquarium trade? b) Where do the fish live? c) What do the fish eat? and d) When is the spawning season of the fish? The questions followed the same order for all respondents.

Table 1 Interview locations in the municipality of Barcelos, middle Negro River basin, Amazonas

\begin{tabular}{lll}
\hline Interview locations & Fishers interviewed (n) & Percentage (\%) \\
\hline City of Barcelos & 52 & 58.4 \\
Ponta da Terra & 8 & 8.9 \\
Santa Inês & 4 & 4.4 \\
Daracuá & 8 & 8.9 \\
Mulufú & 6 & 6.7 \\
Romão & 3 & 3.3 \\
Elesbão & 4 & 4.4 \\
Bacabal & 2 & 2.2 \\
Jaqueira & 2 & 2.2 \\
Total & 89 & 100 \\
\hline
\end{tabular}

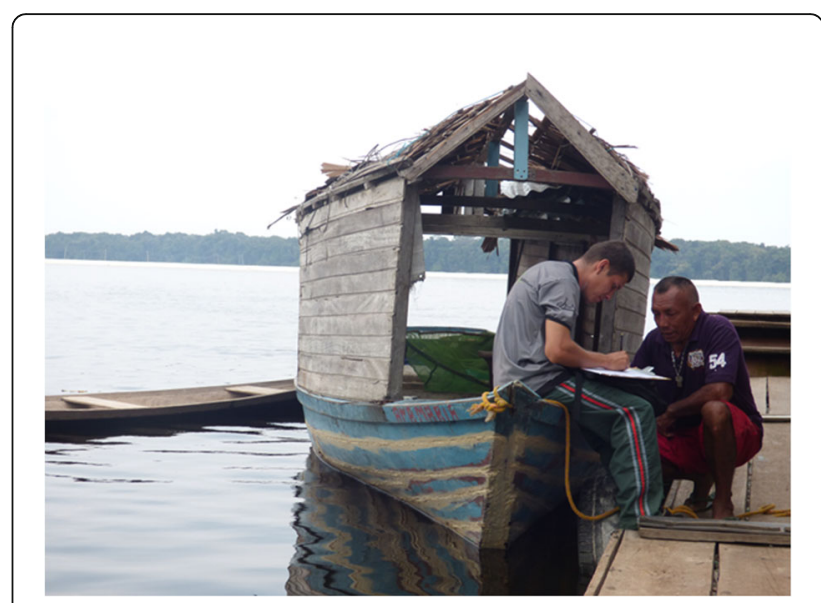

Fig. 2 Interview with the piabeiros

\section{Data analysis}

The data obtained in the interviews were tabulated in spreadsheets and analyzed using descriptive statistics based on the frequency of responses from the fishers. The words and expressions of the fishers were kept in their original form to guarantee the accuracy of the information.

For identification and taxonomic classification of fish species, we compared the descriptions made by the fishers in the interviews with the information contained in the scientific literature and with the FishBase database. Consultations were also held with specialist fishers (experienced in the practice of ornamental fishing) and local fish traders, in order to obtain information related to the ornamental species traded in the region.

Due to the amount and diversity of information cited by fishers during the interviews, we chose to group information into categories for better presentation and discussion. In addition, fishers cited more than one piece of information in a single category (e.g., types of habitats and food aspects), and in this case we chose to record the relative frequency of information for both categories (Table 2).

\section{Results}

\section{Ethnoichthyology of fishers of ornamental fish}

In all, the interviewed fishers cited 41 ethnospecies (popular names) when referring to the species of fish caught and traded in the region for ornamental purposes (Table 3). The categories mentioned corresponded to 10 families and 4 ethnocategories, a group in which several ethnospecies of the same family or genus are aggregated; for example, the group "Araias" is formed by fish of the Potamotrygonidae family (Fig. 3).

The Loricariidae family showed a predominance of ten ethnospecies, especially the bodó seda-Ancistrus dolichopterus (kner, 1854) (6.4\%) and bodó onça- 
Table 2 Categories and variables created based on local ecological knowledge of fishers

\begin{tabular}{ll}
\hline Categories & Data variables \\
\hline Behavioral aspects & $\begin{array}{l}\text { Lives in shoals (species of fish that usually live in the water column in groups with individuals of the same or another } \\
\text { species), solitary (species of fish that do not live in aggregate form), migratory (species of fish that perform migratory } \\
\text { movements) and resident (species of fish generally demersal, considered not making migratory movements) }\end{array}$ \\
Habitat types & $\begin{array}{l}\text { River, lake, beach (sand banks formed in the period when river levels are lowest in the Amazon), stream, flooded forest } \\
\text { (igapó), floodplain, aquatic vegetation, river bank }\end{array}$ \\
Food aspects & $\begin{array}{l}\text { Periphyton (slime), fish, plant material (fruits, flowers, leaves, vegetable debris and grass), worm (Oligochaeta), detritus (sand, } \\
\text { mud, clay and water), insects (spiders and mosquitoes larvae (Diptera)), non-plant prganic material (decaying animal remains, } \\
\text { blood, eggs from other fish), shrimp (Decapoda), crab (Decapoda), snails (Gastropoda) }\end{array}$ \\
Breeding season of & Rising (April to June), full (July to August), ebb (September to November) and dry (December to March) \\
species &
\end{tabular}

Pterygoplichthys gibbiceps (Kner, 1854) (3.8\%). Fishers also mention five species of stingray (Potamotrygonidae), namely araia motoro-Potamotrygon motoro (Müller and Henle, 1841) (3.8\%), araia cururu-Potamotrygon wallacei (Carvalho, Rosa and Araújo, 2016) (3.6\%), araia aiereba-Paratrygon aiereba (Müller and Henle, 1841) (0.95\%), araia schroederi-Potamotrygon schroederi (Fernández-Yépez, 1958) (0.16\%), and araia orbignyiPotamotrygon orbignyi (Castelnau, 1855) (0.16\%). The main ethnospecies mentioned were cardinal-Paracheirodon axelrodi (Schultz, 1956) (12.5\%), rodostomoHemigrammus bleheri (Géry and Mahnert, 1986) (8.3\%), bodó seda-Ancistrus dolichopterus (6.4\%), acará discoSymphysodon discus (5.3\%), and araia motoro-Potamotrygon motoro (3.8\%); together, these represent $36.5 \%$ of all ethnospecies cited by fishers (Table 3) (Fig. 4).

Some ethnospecies were reported only once, such as acará azulão, acará baru, acará peixeiro, araia orbignyi, araia schroederi, bodó cor de mapa, bodó panda, bodó pedra, bodó tuí, borboleta branca, borboleta rajada, farowela, ituí cavalo, and uricaia (Table 3).

We observed that the fishers in the region create clusters of ethnospecies, which we call ethnocategories, called "piabas," bodós, araias, and varieties (a group which includes several ethnospecies from different families and genres) (Fig. 2).

\section{Behavioral aspects of ornamental fish}

According to fishers, the majority of ornamental fish species called "piabas" prefer to live in groups (28.9\%) and make migratory movements (26.1\%) known locally as "arribação," which is an event that consists of the displacement of some species in the seasonal period of the rise or ebb of the waters of the region's rivers, and includes species such as cardinal ( $P$. axelrodi), borboleta (Carnegiella spp.), rodostomo (H. bleheri) and rosacéu (Hyphessobrycon spp.) (Table 4).

In relation to the behavioral aspects mentioned above, we can observe such examples of the behavior of the cardinal ( $P$. axelrodi) according to what was reported by the interviewee, as reproduced below:
[...] They are always swimming in a shoal, the large ones all mixed in with the young (WPS, 34 years old).

Fishers reported that fish they considered being "sedentary" choose to live alone (13.3\%) and do not carry out migratory movements and were considered resident species (16.1\%). The species cited by fishers with such characteristics were mainly araias (Potamotrygonidae), as cururu ( $P$. wallacei) and motoro ( $P$. motoro), in addition to the bodó onça ( $P$. gibbiceps) and bodó seda (A. dolichopterus) (Table 4).

According to fishers, the ornamental fish in this region inhabit different types of environments, which change according to the seasonal regime of the local rivers. In this scenario, streams (34.1\%), locally known as igarapés, were mentioned as the main habitat for ornamental fish species, followed by lakes (7.1\%) and igapós (5.5\%) which are a portion of forests that are flooded by the Negro River's black waters. Aquatic vegetation and riverbanks were the environments that were considered to have the lowest occurrences of the species $(1.4 \%$ and $0.15 \%$ respectively).

Fishers also reported that some environments, such as streams and the igapós, are inhabited by fish species belonging to the piabas (e.g., cardinal-P. axelrodi), rodostomo (H. bleheri), borboleta (Carnegiella spp), and rosacéo (Hyphessobrycon spp) and other varieties (e.g., acará disco-S. discus). For the bodó seda (A. dolichopterus) species, the igarapé was mentioned as the main habitat; however, for the main species belonging to the ethnocategory of araias, the beach and lake environments were the most cited, while the igarapé and lake were related to fish habitats of the ethnocategory bodó (Table 5).

\section{Feeding habits of the ornamental fish}

According to the reports of the fishers, the ornamental fish species in the region have diversified diets; however, slime or periphyton $(42.2 \%)$ is the main component of the diet of most species of piabas, as well as the types of 
Table 3 List of ethnospecies cited in interviews by fishers ("piabeiros"). ( $n=$ number of citations by interviewees)

\begin{tabular}{|c|c|c|c|}
\hline Family & Local name/scientific name & Relative frequency (\%) & Absolute frequency $(n)$ \\
\hline \multirow[t]{2}{*}{ Cichlidae } & Acará disco Symphysodon discus Heckel, 1840 & 5.38 & 34 \\
\hline & Acará azulão ${ }^{a}$ & 0.16 & 1 \\
\hline Cichlidae & Acará baru Uaru amphiacanthoides Heckel, 1840 & 0.16 & 1 \\
\hline Cichlidae & Acará peixeiro ${ }^{a}$ & 0.16 & 1 \\
\hline Lebiasinidae & Anostomo trifasciatus Nannostomus trifasciatus Steindachner, 1876 & 0.32 & 2 \\
\hline Cichlidae & Apistograma Apistogramma spp & 3.96 & 25 \\
\hline Potamotrygonidae & Araia aireba Paratrygon aiereba Müller and Henle, 1841 & 0.95 & 6 \\
\hline Potamotrygonidae & Araia cururu Potamotrygon wallacei Carvalho, Rosa and Araújo, 2016 & 3.64 & 23 \\
\hline Potamotrygonidae & Araia motoro Potamotrygon motoro Müller and Henle, 1841 & 3.80 & 24 \\
\hline Potamotrygonidae & Araia orbignyi Potamotrygon orbignyi Castelnau, 1855 & 0.16 & 1 \\
\hline Potamotrygonidae & Araia Schroederi Potamotrygon schroederi Fernández-Yépez, 1958 & 0.16 & 1 \\
\hline Potamotrygonidae & Araia Potamotrygon spp & 4.91 & 31 \\
\hline Osteoglossidae & Aruanã Osteoglossum spp & 0.32 & 2 \\
\hline \multirow[t]{2}{*}{ Loricariidae } & Bodó cor de mapa Peckoltia spp & 0.16 & 1 \\
\hline & Bodó cutia $^{a}$ & 0.63 & 4 \\
\hline Loricariidae & Bodó espinho Pseudocanthicus spp & 0.79 & 5 \\
\hline Loricariidae & Bodó jauari Loricarridae spp & 0.95 & 6 \\
\hline Loricariidae & Bodó luminol Ancistrus spp & 0.32 & 2 \\
\hline \multirow[t]{3}{*}{ Loricariidae } & Bodó onça Pterygoplichthys gibbiceps Kner, 1854 & 3.80 & 24 \\
\hline & Bodó panda ${ }^{a}$ & 0.16 & 1 \\
\hline & Bodó pedra ${ }^{a}$ & 0.16 & 1 \\
\hline Loricariidae & Bodó percote Peckoltia spp & 1.11 & 7 \\
\hline Loricariidae & Bodó seda Ancistrus dolichopterus Kner, 1854 & 6.49 & 41 \\
\hline \multirow[t]{2}{*}{ Loricariidae } & Bodóa $^{a}$ & 1.42 & 9 \\
\hline & Bodó tuía & 0.16 & 1 \\
\hline Loricariidae & Bodó zebra Peckoltia spp & 0.47 & 3 \\
\hline Gasteropelecidae & Borboleta Carnegiella spp & 9.18 & 58 \\
\hline Gasteropelecidae & Borboleta branca Carnegiella marthae Myers, 1927 & 0.16 & 1 \\
\hline Gasteropelecidae & Borboleta rajada Carnegiella strigata Günther, 1864 & 0.16 & 1 \\
\hline Characidae & Cardinal Paracheirodon axelrodi Schultz, 1956 & 12.50 & 79 \\
\hline Callichthyidae & Coridora Corydoras spp & 0.79 & 5 \\
\hline Loricariidae & Farowela Farlowella spp & 0.16 & 1 \\
\hline Apteronotidae & Ituí cavalo Apteronotus albifrons Linnaeus, 1766 & 0.16 & 1 \\
\hline Lebiasinidae & Lápis Nannostomus spp & 6.65 & 42 \\
\hline Lebiasinidae & Marginatus Nannostomus marginatus Eigenmann, 1909 & 2.53 & 16 \\
\hline Characidae & Neon Paracheirodon innesi Myers, 1936 & 3.64 & 23 \\
\hline Belonidae & Peixe agulha Potamorrhaphis guianensis Jardine, 1843 & 0.32 & 2 \\
\hline Characidae & Rodostomo Hemigrammus bleheri Géry and Mahnert, 1986 & 8.39 & 53 \\
\hline \multirow[t]{2}{*}{ Characidae } & Rosacéu Hyphessobrycon spp & 7.75 & 49 \\
\hline & Uricaia $^{a}$ & 0.16 & 1 \\
\hline Cichlidae & Xadrez Dicrossus spp & 6.80 & 43 \\
\hline Total & & 100 & 632 \\
\hline
\end{tabular}

${ }^{a}$ Species for which it was not possible to carry out the identification based on the description of the interviewees 


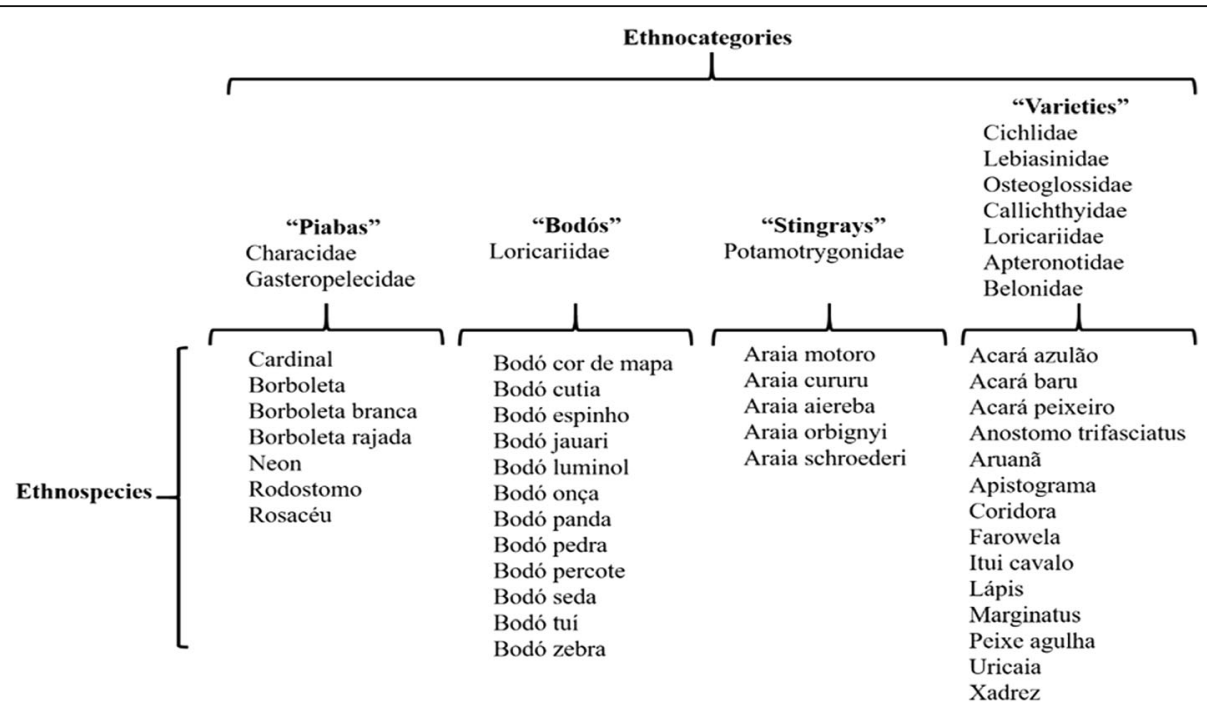

Fig. 3 Ethnocategories of ornamental fish species according to fishers

bodó and some of the other varieties (e.g., acará disco S. discus and lápis Nannostomus spp) (Table 6). In relation to fish with fish-based diets, the majority of fishers associated this habit with fish of the Potamotrygonidae family. However, during the interviews, there were some fishers who also associated the consumption of detritus and earthworms (Table 6) with the fish of this family:

[...] it (the stingray) likes mud and those old leaves from the bottom [...] eats everything, worms, shrimp, crab, fish (HD, 58 years old).
In some cases, fishers linked the consumption of fish to the diet of species such as cardinal ( $P$. axelrodi), rodostomo ( $H$. bleheri), and rosacéu (Hyphessobrycon spp.). Crustaceans, such as shrimp (1.04\%) and crab $(0.58 \%)$, followed by snails $(0.12 \%)$, were less often mentioned (Table 6).

\section{Reproductive aspects of ornamental fish}

For the fishers, the reproductive cycle of the species is directly influenced by the seasonal dynamics of the water levels of the rivers. The period when the river rises $(37.6 \%)$ is the period that they considered to be the main breeding season for most species of ornamental fish in

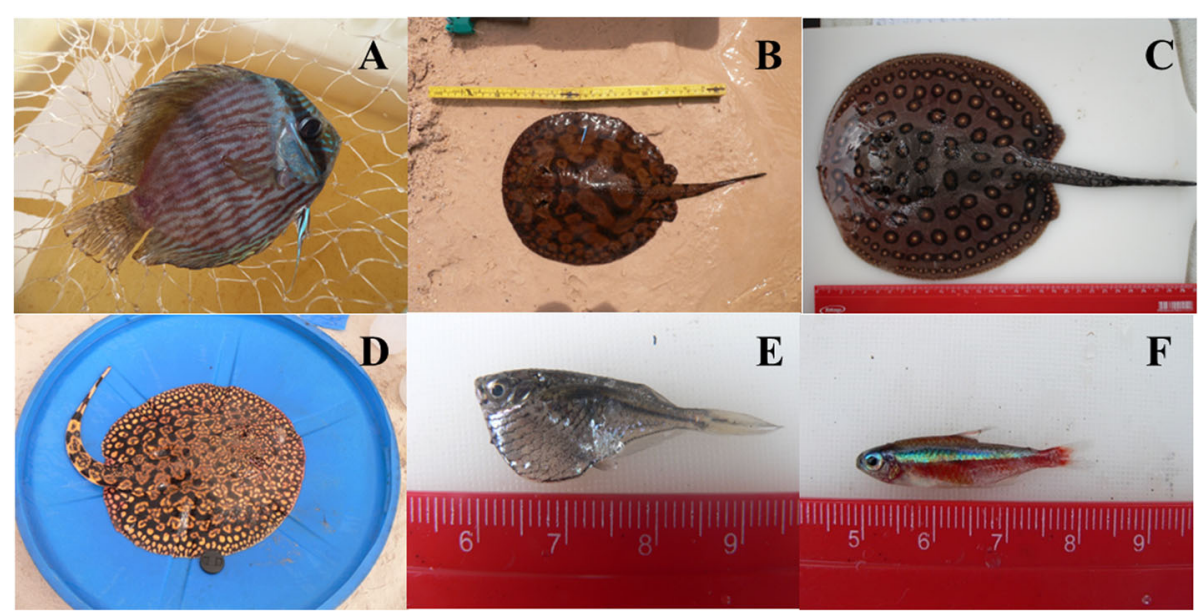

Fig. 4 Some species of ornamental fish reported by piabeiros. (A) Acara disco—Symphysodon discus. (B) Araia cururu—Potamotrygon wallacei. (C) Araia motoro-Potamotrygon motoro. (D) Araia schroederi_Potamotrygon schroederi. (E) Borboleta branca_Carnegiella marthae. (F) Cardinal_-Paracheirodon axelrodi 
Table 4 Behavior of ornamental fish according to the fishers ("piabeiros") of Barcelos, Amazonas

\begin{tabular}{|c|c|c|c|c|c|c|c|c|c|}
\hline \multirow[t]{2}{*}{ Species } & \multicolumn{2}{|c|}{ Solitary } & \multicolumn{2}{|c|}{ Lives in shoals } & \multicolumn{2}{|c|}{ Resident } & \multicolumn{2}{|c|}{ Migratory } & \multirow{2}{*}{$\begin{array}{l}\text { No } \\
\text { answer }\end{array}$} \\
\hline & $\%$ & $n$ & $\%$ & $\mathbf{N}$ & $\%$ & $\mathbf{N}$ & $\%$ & $n$ & \\
\hline Acará disco & 0.68 & 1 & 6.62 & 21 & 2.82 & 5 & 5.94 & 17 & 12 \\
\hline Acará azulão & 0.68 & 1 & & & 0.56 & 1 & & & \\
\hline Acará baru & 0.68 & 1 & & & 0.56 & 1 & & & \\
\hline Acará peixeiro & 0.68 & 1 & & & 0.56 & 1 & & & \\
\hline Anostomo trifaciato & & & 0.32 & 1 & & & 0.35 & 1 & 1 \\
\hline Apistograma & 0.68 & 1 & 2.21 & 7 & 1.69 & 3 & 1.75 & 5 & 17 \\
\hline Araia aireba & 2.74 & 4 & 0.63 & 2 & 2.82 & 5 & 0.35 & 1 & \\
\hline Araia cururu & 13.01 & 19 & 0.95 & 3 & 9.60 & 17 & 1.75 & 5 & 1 \\
\hline Araia motoro & 13.01 & 19 & 0.95 & 3 & 11.30 & 20 & 0.70 & 2 & 2 \\
\hline Araia orbignyi & & & 0.32 & 1 & & & 0.35 & 1 & \\
\hline Araia schroederi & & & 0.32 & 1 & & & 0.35 & 1 & \\
\hline Araia & 16.44 & 24 & 0.63 & 2 & 10.73 & 19 & 2.45 & 7 & 5 \\
\hline Aruanã & & & 0.32 & 1 & & & 0.35 & 1 & 1 \\
\hline Bodó cor de mapa & & & & & & & & & 1 \\
\hline Bodó cutia & 0.68 & 1 & 0.32 & 1 & 0.56 & 1 & 0.35 & 1 & 2 \\
\hline Bodó espinho & 2.05 & 3 & & & 1.69 & 3 & & & 2 \\
\hline Bodó jauari & 2.74 & 4 & & & 2.26 & 4 & & & 2 \\
\hline Bodó luminol & 0.68 & 1 & & & 0.56 & 1 & & & 1 \\
\hline Bodó onça & 11.64 & 17 & 0.63 & 2 & 7.91 & 14 & 1.75 & 5 & 5 \\
\hline Bodó panda & & & & & & & & & 1 \\
\hline Bodó pedra & & & & & & & & & 1 \\
\hline Bodó percote & 3.42 & 5 & & & 2.82 & 5 & & & 2 \\
\hline Bodó seda & 17.12 & 25 & 2.84 & 9 & 15.25 & 27 & 2.45 & 7 & 7 \\
\hline Bodó & 4.11 & 6 & 0.63 & 2 & 3.95 & 7 & 0.35 & 1 & 1 \\
\hline Bodó tui & 0.68 & 1 & & & 0.56 & 1 & & & \\
\hline Bodó zebra & 0.68 & 1 & & & & & 0,35 & 1 & 2 \\
\hline Borboleta & 1.37 & 2 & 13.88 & 44 & 6.78 & 12 & 11.89 & 34 & 12 \\
\hline Borboleta branca & & & 0.32 & 1 & & & 0.35 & 1 & \\
\hline Borboleta rajada & & & 0.32 & 1 & & & 0.35 & 1 & \\
\hline Cardinal & & & 23.03 & 73 & 1.69 & 3 & 24.48 & 70 & 6 \\
\hline Coridora & & & 0.95 & 3 & 0.56 & 1 & 0.70 & 2 & 2 \\
\hline Farowela & & & 0.32 & 1 & & & 0.35 & 1 & \\
\hline Itui cavalo & & & & & & & & & 1 \\
\hline Lápis & 0.68 & 1 & 5.99 & 19 & 2.82 & 5 & 5.24 & 15 & 22 \\
\hline Marginata & & & 2.52 & 8 & & & 2.80 & 8 & 8 \\
\hline Neon & & & 5.99 & 19 & 1.13 & 2 & 5.94 & 17 & 4 \\
\hline Peixe agulha & & & & & & & & & 2 \\
\hline Rodostomo & 2.74 & 4 & 11.36 & 36 & 4.52 & 8 & 11.19 & 32 & 13 \\
\hline Rosacéu & 2.05 & 3 & 10.41 & 33 & 3.95 & 7 & 10.14 & 29 & 13 \\
\hline Uricaia & & & 0.32 & 1 & & & 0.35 & 1 & \\
\hline Xadrez & 0.68 & 1 & 6.94 & 22 & 2.26 & 4 & 6.64 & 19 & 20 \\
\hline Total & 100 & 146 & 100 & 317 & 100 & 177 & 100 & 286 & 169 \\
\hline
\end{tabular}


Table 5 Types of habitats of ornamental fish mentioned by artisanal fishers ("piabeiros")

\begin{tabular}{|c|c|c|c|c|c|c|c|c|c|c|c|c|c|c|c|c|c|}
\hline \multirow[t]{3}{*}{ Species } & \multicolumn{16}{|c|}{ Habitat types } & \multirow{3}{*}{$\begin{array}{l}\text { No } \\
\text { answer }\end{array}$} \\
\hline & \multicolumn{2}{|c|}{ River } & \multicolumn{2}{|l|}{ Lake } & \multicolumn{2}{|c|}{ Stream } & \multicolumn{2}{|c|}{ Beach } & \multicolumn{2}{|c|}{ Forest igapó } & \multicolumn{2}{|c|}{ Flood plain lake } & \multicolumn{2}{|c|}{ Aquatic vegetation } & \multicolumn{2}{|c|}{ River bank } & \\
\hline & $\%$ & $\mathrm{~N}$ & $\%$ & $\mathbf{N}$ & $\%$ & $\mathrm{n}$ & $\%$ & $\mathrm{n}$ & $\%$ & $\mathrm{n}$ & $\%$ & $\mathrm{n}$ & $\%$ & $\mathrm{n}$ & $\%$ & $n$ & \\
\hline Acará disco & 5 & 1 & 2.08 & 1 & 6.99 & 16 & & & 13.51 & 5 & & & & & & & 11 \\
\hline Acará azulão & & & & & 0.44 & 1 & & & & & & & & & & & \\
\hline Acará baru & & & & & 0.44 & 1 & & & & & & & & & & & \\
\hline Acará peixeiro & & & & & 0.44 & 1 & & & & & & & & & & & \\
\hline Anostomo trifaciato & & & 2.8 & 1 & & & & & & & & & & & & & 1 \\
\hline Apistograma & 5 & 1 & 2.8 & 1 & 2.62 & 6 & & & 5.41 & 2 & & & & & & & 17 \\
\hline Araia areba & & & 4.17 & 2 & & & 5.88 & 1 & & & & & & & & & 3 \\
\hline Araia cururu & & & 6.25 & 3 & 2.62 & 6 & 17.65 & 3 & & & & & & & & & 12 \\
\hline Araia motoro & & & 6.25 & 3 & 3.06 & 7 & 23.53 & 4 & & & & & & & & & 11 \\
\hline Araia orbignyi & & & & & & & 5.88 & 1 & & & & & & & & & \\
\hline Araia schroederi & & & & & & & 5.88 & 1 & & & & & & & & & \\
\hline Araia & 15 & 3 & 2.08 & 1 & 9.17 & 21 & & & & & & & 10 & 1 & & & 8 \\
\hline Aruanã & 5 & 1 & & & & & & & & & & & & & & & 1 \\
\hline Bodó cor de mapa & & & & & 0.44 & 1 & & & & & & & & & & & \\
\hline Bodó cutia & & & 4.17 & 2 & 0.87 & 2 & 5.88 & 1 & & & & & & & & & 1 \\
\hline Bodó espinho & & & 2.08 & 1 & 0.87 & 2 & & & 2.70 & 1 & & & & & & & 1 \\
\hline Bodó jauari & & & 2.08 & 1 & 1.31 & 3 & 5.88 & 1 & 2.70 & 1 & & & & & & & 2 \\
\hline Bodó luminol & & & 2.08 & 1 & 0.44 & 1 & 5.88 & 1 & & & & & & & & & 1 \\
\hline Bodó onça & & & 4.17 & 2 & 3.06 & 7 & & & 8.11 & 3 & & & & & & & 12 \\
\hline Bodó panda & & & & & 0.44 & 1 & & & & & & & & & & & \\
\hline Bodó pedra & & & & & & & & & & & & & & & & & 1 \\
\hline Bodó percote & & & 2.08 & 1 & 0.44 & 1 & 11.76 & 2 & 2.70 & 1 & & & & & 100 & 1 & 3 \\
\hline Bodó seda & & & 4.17 & 2 & 6.55 & 15 & & & 18.92 & 7 & & & & & & & 17 \\
\hline Bodó & & & & & 2.62 & 6 & & & & & & & & & & & 3 \\
\hline Bodó tui & & & & & & & & & & & & & & & & & 1 \\
\hline Bodó zebra & & & 2.08 & 1 & 0.44 & 1 & & & & & & & & & & & 1 \\
\hline Borboleta & 10 & 2 & 6.25 & 3 & 6.99 & 16 & 11.76 & 2 & 8.11 & 3 & 12.50 & 2 & & & & & 34 \\
\hline Borboleta branca & & & 2.08 & 1 & & & & & & & & & & & & & \\
\hline Borboleta rajada & & & 2.08 & 1 & & & & & & & & & & & & & \\
\hline Cardinal & 15 & 3 & 16.70 & 8 & 17.03 & 39 & & & 8.11 & 3 & 25 & 4 & 20 & 2 & & & 26 \\
\hline Coridora & & & 2.08 & 1 & 0.87 & 2 & & & & & & & & & & & 2 \\
\hline Farowela & & & & & 0.44 & 1 & & & & & & & & & & & \\
\hline Itui cavalo & & & & & & & & & & & & & & & & & 1 \\
\hline Lápis & 5 & 1 & 4.17 & 2 & 5.24 & 12 & & & 2.70 & 1 & 12.50 & 2 & & & & & 25 \\
\hline Marginata & 10 & 2 & 2.08 & 1 & 1.75 & 4 & & & & & & & 10 & 1 & & & 10 \\
\hline Neon & 5 & 1 & 2.08 & 1 & 4.37 & 10 & & & 8.11 & 3 & 6.25 & 1 & & & & & 9 \\
\hline Peixe agulha & & & & & & & & & & & & & & & & & 2 \\
\hline Rodostomo & 10 & 2 & & & 7.86 & 18 & & & & & 25 & 4 & 60 & 6 & & & 25 \\
\hline Rosacéu & 10 & 2 & 10.4 & 5 & 6.55 & 15 & & & 10.81 & 4 & 18.75 & 3 & & & & & 23 \\
\hline Uricaia & & & & & 0.44 & 1 & & & & & & & & & & & \\
\hline Xadrez & 5 & 1 & 4.17 & 2 & 5.24 & 12 & & & 8.11 & 3 & & & & & & & 28 \\
\hline Total & 100 & 20 & 100 & 48 & 100 & 229 & 100 & 17 & 100 & 37 & 100 & 16 & 100 & 10 & 100 & 1 & 292 \\
\hline
\end{tabular}


Table 6 Feeding habits of ornamental fish according to artisanal fishers ("piabeiros")

\begin{tabular}{|c|c|c|c|c|c|c|c|c|c|c|c|c|c|c|c|c|c|c|c|c|c|}
\hline \multirow[t]{3}{*}{ Species } & \multicolumn{20}{|c|}{ Ornamental fish food items } & \multirow{3}{*}{$\begin{array}{l}\text { No } \\
\text { answer }\end{array}$} \\
\hline & \multicolumn{2}{|c|}{$\begin{array}{l}\text { Shrimp } \\
\text { Decapoda }\end{array}$} & \multicolumn{2}{|c|}{$\begin{array}{l}\text { Snails } \\
\text { Gastropoda }\end{array}$} & \multicolumn{2}{|c|}{$\begin{array}{l}\text { Crab } \\
\text { Decapoda }\end{array}$} & \multicolumn{2}{|c|}{ Insects } & \multicolumn{2}{|c|}{$\begin{array}{l}\text { Worm } \\
\text { Oligochaeta }\end{array}$} & \multicolumn{2}{|l|}{ Fish } & \multicolumn{2}{|c|}{ Detritus } & \multicolumn{2}{|c|}{ Periphyton } & \multicolumn{2}{|c|}{$\begin{array}{l}\text { Plant } \\
\text { material }\end{array}$} & \multicolumn{2}{|c|}{$\begin{array}{l}\text { Non- } \\
\text { plant } \\
\text { organic } \\
\text { material }\end{array}$} & \\
\hline & $\%$ & $\mathbf{n}$ & $\%$ & $\mathbf{N}$ & $\%$ & $\mathbf{N}$ & $\%$ & $\mathrm{n}$ & $\%$ & $\mathbf{n}$ & $\%$ & $\mathbf{N}$ & $\%$ & $\mathbf{n}$ & $\%$ & $\mathbf{n}$ & $\%$ & $\mathbf{n}$ & $\%$ & $\mathbf{n}$ & \\
\hline Acará disco & & & & & & & 4.76 & 1 & & & 2.27 & 2 & & & 8.22 & 30 & 3.08 & 2 & 5.88 & 1 & 4 \\
\hline Acará azulão & & & & & & & & & & & & & & & 0.27 & 1 & 1.54 & 1 & & & \\
\hline Acará baru & & & & & & & & & & & & & & & 0.27 & 1 & 1.54 & 1 & & & \\
\hline Acará peixeiro & & & & & & & & & & & & & & & 0.27 & 1 & 1.54 & 1 & & & \\
\hline $\begin{array}{l}\text { Anostomo } \\
\text { trifaciato }\end{array}$ & & & & & & & & & & & & & & & 0.55 & 2 & & & & & \\
\hline Apistograma & & & & & & & & & 2 & 1 & 1.14 & 1 & & & 3.01 & 11 & 3.08 & 2 & 5.88 & 1 & 14 \\
\hline Araia aireba & 22.22 & 2 & & & 20 & 1 & & & 6 & 3 & 2.27 & 2 & 7.69 & 2 & 0.27 & 1 & & & 5.88 & 1 & 1 \\
\hline Araia cururu & 33.33 & 3 & & & 60 & 3 & & & 32 & 16 & 11.4 & 10 & 19.23 & 5 & 0.27 & 1 & 3.08 & 2 & 5.88 & 1 & 7 \\
\hline Araia motoro & 22.22 & 2 & 100 & 1 & 20 & 1 & & & 32 & 16 & 11.4 & 10 & 3.85 & 1 & 0.27 & 1 & 1.54 & 1 & & & 8 \\
\hline Araia orbignyi & & & & & & & & & & & 1.14 & 1 & & & & & & & & & \\
\hline Araia schroederi & & & & & & & & & & & 1.14 & 1 & & & & & & & & & \\
\hline Araia & 22.22 & 2 & & & & & & & 28 & 14 & 17 & 15 & 30.77 & 8 & 1.10 & 4 & & & & & 16 \\
\hline Aruanã & & & & & & & & & & & 1.14 & 1 & & & & & & & & & 1 \\
\hline $\begin{array}{l}\text { Bodó cor de } \\
\text { mapa }\end{array}$ & & & & & & & & & & & & & & & 0.27 & 1 & & & & & \\
\hline Bodó cutia & & & & & & & & & & & & & 3.85 & 1 & 1.10 & 4 & & & & & \\
\hline Bodó espinho & & & & & & & 4.76 & 1 & & & & & & & 1.10 & 4 & & & & & \\
\hline Bodó jauari & & & & & & & & & & & & & 3.85 & 1 & 1.64 & 6 & & & & & \\
\hline Bodó luminol & & & & & & & & & & & & & 3.85 & 1 & 0.55 & 2 & & & & & \\
\hline Bodó onça & & & & & & & 4.76 & 1 & & & & & 7.69 & 2 & 5.48 & 20 & & & & & 4 \\
\hline Bodó panda & & & & & & & & & & & & & & & 0.27 & 1 & & & & & \\
\hline Bodó pedra & & & & & & & & & & & & & & & 0.27 & 1 & & & & & \\
\hline Bodó percote & & & & & & & & & & & & & 3.85 & 1 & 1.92 & 7 & & & & & \\
\hline Bodó seda & & & & & & & 4.76 & 1 & & & & & 7.69 & 2 & 10.14 & 37 & & & & & 4 \\
\hline Bodó & & & & & & & & & & & & & & & 1.92 & 7 & & & & & 2 \\
\hline Bodó tui & & & & & & & & & & & & & & & 0.27 & 1 & & & & & \\
\hline Bodó zebra & & & & & & & & & & & & & & & 0.82 & 3 & & & & & \\
\hline Borboleta & & & & & & & 38.10 & 8 & & & 2.27 & 2 & 3.85 & 1 & 8.49 & 31 & 13.85 & 9 & 5.88 & 1 & 27 \\
\hline Borboleta branca & & & & & & & & & & & & & & & 0.27 & 1 & & & & & \\
\hline Borboleta rajada & & & & & & & & & & & & & & & 0.27 & 1 & & & & & \\
\hline Cardinal & & & & & & & 19.05 & 4 & & & 21.6 & 19 & & & 13.42 & 49 & 30.77 & 20 & 17.65 & 3 & 30 \\
\hline Coridora & & & & & & & & & & & & & & & 1.10 & 4 & & & & & 1 \\
\hline Farowela & & & & & & & & & & & & & & & & & & & & & 1 \\
\hline Itui cavalo & & & & & & & & & & & & & & & 0.27 & 1 & & & & & \\
\hline Lápis & & & & & & & & & & & & & & & 5.48 & 20 & 4.62 & 3 & 5.88 & 1 & 22 \\
\hline Marginata & & & & & & & 4.76 & 1 & & & 1.14 & 1 & & & 2.19 & 8 & 3.08 & 2 & & & 8 \\
\hline Neon & & & & & & & & & & & 3.41 & 3 & & & 4.11 & 15 & 3.08 & 2 & & & 8 \\
\hline Peixe agulha & & & & & & & & & & & & & & & 0.55 & 2 & & & & & \\
\hline Rodostomo & & & & & & & 14.29 & 3 & & & 11.4 & 10 & & & 8.22 & 30 & 12.31 & 8 & 11.76 & 2 & 23 \\
\hline Rosacéu & & & & & & & 4.76 & 1 & & & 9.09 & 8 & & & 8.77 & 32 & 9.23 & 6 & 29.41 & 5 & 17 \\
\hline
\end{tabular}


Table 6 Feeding habits of ornamental fish according to artisanal fishers ("piabeiros") (Continued)

\begin{tabular}{|c|c|c|c|c|c|c|c|c|c|c|c|c|c|c|c|c|c|c|c|c|c|}
\hline \multirow[t]{3}{*}{ Species } & \multicolumn{20}{|c|}{ Ornamental fish food items } & \multirow{3}{*}{$\begin{array}{l}\text { No } \\
\text { answer }\end{array}$} \\
\hline & \multicolumn{2}{|c|}{$\begin{array}{l}\text { Shrimp } \\
\text { Decapoda }\end{array}$} & \multicolumn{2}{|c|}{$\begin{array}{l}\text { Snails } \\
\text { Gastropoda }\end{array}$} & \multicolumn{2}{|c|}{$\begin{array}{l}\text { Crab } \\
\text { Decapoda }\end{array}$} & \multicolumn{2}{|c|}{ Insects } & \multicolumn{2}{|c|}{$\begin{array}{l}\text { Worm } \\
\text { Oligochaeta }\end{array}$} & \multicolumn{2}{|c|}{ Fish } & \multicolumn{2}{|c|}{ Detritus } & \multicolumn{2}{|c|}{ Periphyton } & \multicolumn{2}{|c|}{$\begin{array}{l}\text { Plant } \\
\text { material }\end{array}$} & \multicolumn{2}{|c|}{$\begin{array}{l}\text { Non- } \\
\text { plant } \\
\text { organic } \\
\text { material }\end{array}$} & \\
\hline & $\%$ & $\mathrm{n}$ & $\%$ & $\mathbf{N}$ & $\%$ & $\mathrm{~N}$ & $\%$ & $\mathrm{n}$ & $\%$ & $\mathrm{n}$ & $\%$ & $\mathrm{~N}$ & $\%$ & $\mathrm{n}$ & $\%$ & $\mathrm{n}$ & $\%$ & $\mathrm{n}$ & $\%$ & $\mathrm{n}$ & \\
\hline Uricaia & & & & & & & & & & & & & & & & & & & & & 1 \\
\hline Xadrez & & & & & & & & & & & 2.27 & 2 & 3.85 & 1 & 6.58 & 24 & 7.69 & 5 & 5.88 & 1 & 19 \\
\hline Total & 100 & 9 & 100 & 1 & 100 & 5 & 100 & 21 & 100 & 50 & 100 & 88 & 100 & 26 & 100 & 365 & 100 & 65 & 100 & 17 & 218 \\
\hline
\end{tabular}

the region (Table 7), as demonstrated by the fishers' knowledge reported below:

[...] Every ornamental fish has offspring in the flood and only migrates in the "arribação," when they disappear (SCP, 45 years).

The fishers attributed the periods of ebb and when the river is full as periods of reproduction of the species of the ethnocategory araia (Potamotrygonidae) and bodó (Loricariidae) (Table 7). Some fishers demonstrated that they were unaware of the reproductive aspects of some species of local ornamental fish, as shown in excerpts from the following interviews:

[...] For the cardinal, we only find them with eggs (mature oocytes) at the time of the migratory season (species migration period). I think it has no male, because we never saw a cardinal in that period that did not have eggs (JNG, 46 years old).

[...] The cardinal spawns the young, do you know why? We only see the young - we do not see the eggs (ACL, 56 years old).

[...] You know, I've never seen this fish with young (RRS, 51 years old) (fisherman referring to the Borboleta (Carnegiella spp.).

\section{Discussion}

The species and ornamental fish families described by the fishers in our study are among the most commercialized in the middle region of the Negro River basin $[13,15,18,19]$.

Like the cardinal, the fishers cited other ethnospecies that make up a large portion of the fish exported from the Amazon, such as rodostomo $(H$. bleheri), borboleta (Carnegiella spp.), acará disco (S. discus), and ethnocategories araias (Potamotrygonidae) and bodó (Loricariidae). Rodostomo ranks second on the list of the species most exported from the state, second only to the cardinal, and corresponds to about $6 \%$ of the total volume of fish exported (20 to 30 million fish exported annually from the state of Amazonas) [25].

According to [14], ornamental stingray fishing in the middle region of the Negro River basin is already a consolidated activity, and since the regulation of fishing and export of freshwater stingrays (quota system) came into force in 1998, approximately 130,000 rays have been exported from the region, the main species being $P$. motoro, P. wallacei, P. schroederi, P. orbignyi, Potamotrygon leopoldi (Castex and Castello, 1970), and Potamotrygon henlei (Castelnau, 1855).

Artisanal fishers from the middle Negro River basin demonstrated their knowledge of a vast diversity of species and presented their own way of classifying local fish through the formation of ethnocategories. In Brazil, several studies have sought to investigate the different ways of classifying fish caught by artisanal fishers, demonstrating that fishers make groupings of species in a hierarchical manner, based on morphological, behavioral, or ecological criteria $[6,16]$.

Some fish species in Table 3 depicted the absolute frequency of only one. This low representation is due to the enormous diversity that occurs in the locality, as well as the difficulty that the fisher has in classifying some species that were portrayed in this study, due to the similarity that they share with other species.

According to Begossi et al. [26], riverine fishers from the Amazon usually seek to identify fish species by their similarities in terms of morphology, diet, habitat, or behavior, classifying them as "cousins" or "relatives." However, in our study, it is something that needs to be investigated through future studies in which such aspects are more thoroughly investigated.

Based on the fishers' reports, it can be seen that the behavior, diet, and reproduction of ornamental fish species are related to seasonal fluctuations of the rivers in the region. Studies show that the seasonal variations in the water level of the Negro River favor the emergence of new habitats, such as lakes, temporary beaches, flooded fields, and igapós (flooded forests), which serve as a shelter and breeding and feeding grounds for aquatic communities, and such changes end up directly influencing the composition of the ichthyofauna $[27,28]$. 
Table 7 Reproduction period of ornamental fish according to artisanal fishers ("piabeiros")

\begin{tabular}{|c|c|c|c|c|c|c|c|c|c|}
\hline \multirow[t]{3}{*}{ Species } & \multicolumn{8}{|c|}{ Reproductive seasons } & \multirow{3}{*}{$\begin{array}{l}\text { No } \\
\text { answer }\end{array}$} \\
\hline & \multicolumn{2}{|c|}{ Rising } & \multicolumn{2}{|l|}{ Full } & \multicolumn{2}{|l|}{ Ebb } & \multicolumn{2}{|l|}{ Low } & \\
\hline & $\%$ & $\mathbf{n}$ & $\%$ & $\mathrm{~N}$ & $\%$ & $\mathbf{n}$ & $\%$ & $\mathbf{n}$ & \\
\hline Acará disco & 5.11 & 12 & 2.38 & 1 & 10.45 & 7 & 17.39 & 4 & 10 \\
\hline Acará azulão & & & & & 1.49 & 1 & & & \\
\hline Acará baru & & & & & 1.49 & 1 & & & \\
\hline Acará peixeiro & & & & & 1.49 & 1 & & & \\
\hline Anostomo trifaciato & & & & & & & & & 2 \\
\hline Apistograma & 2.55 & 6 & 4.76 & 2 & 2.99 & 2 & & & 15 \\
\hline Araia aireba & & & & & & & & & 6 \\
\hline Araia cururu & 0.43 & 1 & & & 2.99 & 2 & & & 20 \\
\hline Araia motoro & & & & & 2.99 & 2 & 4.35 & 1 & 21 \\
\hline Araira orbignyi & & & & & & & & & 1 \\
\hline Araia schroederi & & & & & & & & & 1 \\
\hline Araia & 3.40 & 8 & 2.38 & 1 & 19.40 & 13 & 39.13 & 9 & \\
\hline Aruanã & 0.43 & 1 & & & & & & & 1 \\
\hline Bodó cor de mapa & & & & & & & & & 1 \\
\hline Bodó cutia & 0.85 & 2 & & & & & & & 2 \\
\hline Bodó espinho & 0.43 & 1 & & & 1.49 & 1 & & & 3 \\
\hline Bodó jauari & & & & & 1.49 & 1 & & & 5 \\
\hline Bodó luminol & & & & & & & & & 2 \\
\hline Bodó onça & 2.13 & 5 & & & 4.48 & 3 & & & 8 \\
\hline Bodó panda & & & & & & & & & 1 \\
\hline Bodó pedra & & & & & & & & & 1 \\
\hline Bodó percote & & & & & & & & & 7 \\
\hline Bodó seda & 3.83 & 9 & 4.76 & 2 & 11.94 & 8 & & & 22 \\
\hline Bodó & & & & & 2.99 & 2 & 26.09 & 6 & 1 \\
\hline Bodó tui & & & & & & & & & 1 \\
\hline Bodó zebra & 0.43 & 1 & & & & & & & 2 \\
\hline Borboleta & 9.79 & 23 & 7.14 & 3 & 4.48 & 3 & 4.35 & 1 & 28 \\
\hline Borboleta branca & & & & & & & & & 1 \\
\hline Borboleta rajada & & & & & & & & & 1 \\
\hline Cardinal & 23.83 & 56 & 35.71 & 15 & 4.48 & 3 & 4.35 & 1 & 4 \\
\hline Coridora & 0.43 & 1 & & & 2.99 & 2 & & & 2 \\
\hline Farowela & 0.43 & 1 & & & & & & & \\
\hline Itui cavalo & & & & & & & & & 1 \\
\hline Lápis & 6.38 & 15 & 7.14 & 3 & 4.48 & 3 & & & 21 \\
\hline Marginata & 2.98 & 7 & 2.38 & 1 & 1.49 & 1 & & & 7 \\
\hline Neon & 6.38 & 15 & 4.76 & 2 & 2.99 & 2 & & & 4 \\
\hline Peixe agulha & & & & & & & & & 2 \\
\hline Rodostomo & 10.21 & 24 & 14.29 & 6 & 4.48 & 3 & 4.35 & 1 & 19 \\
\hline Rosacéu & 11.91 & 28 & 7.14 & 3 & 4.48 & 3 & & & 15 \\
\hline Uricaia & 0.43 & 1 & & & & & & & \\
\hline Xadrez & 7.66 & 18 & 7.14 & 3 & 4.48 & 3 & & & 19 \\
\hline Total & 100 & 235 & 100 & 42 & 100 & 67 & 100 & 23 & 257 \\
\hline
\end{tabular}


In the study by [29], in the region of the Anavilhanas National Park (middle Negro River region), the authors demonstrate how the hydrological cycle influences the composition and structure of fish assemblages in the local lakes and igapós, and the important role of igapós in maintaining the diversity and abundance of ichthyofauna in black waters is also emphasized.

Fish species of the ethnocategory "piabas" in their majority were considered by fishers as species that present behavior of coexistence in groups, and in the period of the floods of the rivers, they carry out migratory movements locally known as "arribação" (migration). Such behavior of the species of this group described by the fishers is consistent with that reported in other studies carried out in the middle region of the Negro River basin [30]. Formations of aggregations by species of ornamental fish have also been described in other regions of the Amazon, as is the case of the discus (S. aequifasciatus) in the lower Solimões river and lower Purus river in the Amazon, where in the dry season (September to November), schools of fish are concentrated around submerged tree branches on the banks of lakes and rivers [12,31].

The differences between the types of waters in the rivers of the Amazon basin may influence the distribution of freshwater stingrays of the Potamotrygonidae family and act as a hydrological filter for the dispersion of fish species in this group [32]. In the study developed by [33], the authors observed ecophysiological differentiations in the preferences of environments between the species of rays (P. motoro, $P$. wallacei, and $P$. aiereba) in the middle of the Negro River region. Artisanal fishers from Barcelos and the riverine communities of the Negro River report that the local freshwater stingrays have a preference for lake areas, beaches, and rivers, which are the same habitats cited by fishers in [17]. The information described in the aforementioned studies may help us to understand the fact that the fishers consider the fish belonging to this ethnocategory as residents, since the species have different distribution patterns.

The streams in the region known as "igarapés" were identified as the main places where the species of ornamental fish live. This is the case of the Acará disco $S$. discus, a species from the lower regions of the Negro, Trombetas, and Abacaxis Rivers, for which streams (igarapés) are the main habitat [34]. This environment is also seen as the main artisanal fishing ground for ornamental fish in the region $[15,19]$.

The flood period favors a greater availability of food and shelter for fish, since there is a greater availability of space, for example, the igapó in the forest that appears seasonally [28]. According to fishers, the diet of ornamental fish species is quite diverse; however, periphyton was identified as the main food item of most species. It was observed that the ornamental fish species from the middle Negro River basin that were cited by fishers tend to inhabit igarapés and igapó forests at different times and occupy these environments in search of food [25, 30]. According to [35], the food base for some of the fish in the igarapé is composed of small terrestrial invertebrates (ants and termites) when they fall into the water, as well as the mosquito larvae (Diptera) present at the bottom of these environments.

Regarding the diet of the species, fishers reported that fish in the Potamotrygonidae family have a diet based on fish, in addition to shrimp, crab, and snail. According to [36], who analyzed the stomach contents of four species of freshwater stingrays ( $P$. motoro, $P$. orbignyi, $P$. wallacei and $P$. aiereba) from the middle of the Negro River basin, it was observed that the diet of these stingrays was basically composed of fish, crustaceans, and insects. However, there are differences in proportions, possibly due to the different types of uses of microhabitats and foraging substrates of these fish. Feeding behavior and morphological characteristics can also influence the composition of the diet of these animals [37].

Some fishers reported that fish of the ethnocategory piabas (cardinal, rodostomo, and rosacéu) feed on fish, while fish of the ethnocategory araias fed on debris (mud, decomposing sand, and leaves) and earthworms. In the first case, such placement may be related to the type of food offered to catch fish, where the caught fish are kept for a certain period in ponds built with screens on the banks of the rivers by the "piabeiros" and fed with cooked fish meat, as observed in the field. While the statement that araias feed on earthworms and debris may be linked to the fact that these animals are partly associated with the substrate of rivers, lakes, streams, and beaches, between sand and mud, and this may have led fishers to relate this behavior to the eating habits of these fish.

Some species of fish in the Amazon show spawning synchronism with changes in the hydrological cycle of rivers and annual rainfall regimes and perform migrations called "piracema" [38]. As well as their feeding behavior, in the fishers' view, the reproductive cycle of ornamental fish species is also influenced by annual variations in water levels in the region's rivers, with the period of rising river levels being the main breeding season for the species. In the Amazon region, several species of ornamental fish demonstrate synchronism of the reproductive cycle with the initial phase of increasing water levels [25, 30, 31].

\section{Conclusions}

In summary, our study demonstrated that the fishers of ornamental fish in the middle region of the Negro River 
basin, who are known as "piabeiros," possess important information related to the behavioral, dietary, and reproductive aspects of local ornamental fish species. Although our methods are based on descriptive analysis and this may limit our conclusions, we believe that our number of respondents poses a representative sample of fishers and their ecological knowledge on local species.

We hope that our study will contribute to the emergence of new studies aimed at understanding the local ecological knowledge of fishers of ornamental fish species in the Amazon, since the existing information on the topic is still incipient. The information presented may assist in the decision-making process for the management of local fishery resources and contribute to the resumption of growth and sustainability of the capture of ornamental fish.

\section{Acknowledgements}

We would like to thank the Coordenação de Aperfeicoamento de Pessoal de Nível Superior (CAPES) - Financial Code 001, and the Fundação Araucária for a scholarship granted to the first author. A. T. Oliveira has a research fellowship from CNPq/Brazil.

\section{Authors' contributions}

All authors contributed equally to this work. All authors wrote, reviewed, and commented on the manuscript. All authors have read and agreed to the published version of the manuscript.

\section{Funding}

This study was funded by the Fundação de Amparo à Pesquisa do Estado do Amazonas (FAPEAM), through the Propesca Project - Rio Negro (Call 010/14, process 062.00933/2015) and PAPAC (Call 05/2019, process 062.00847/2019); Instituto Federal de Educação, Ciência e Tecnologia do Amazonas - IFAM.

\section{Availability of data and materials}

All data generated or analyzed during this study are included in this published article.

\section{Declarations}

Ethics approval and consent to participate

All interviews conformed to the International Society of Ethnobiology's Code of Ethics. The Scientific Research and Ethical Committee at the Centro Universitário Nilton Lins (Issue date-no.: August 24, 2017-Number 2.238.505) approved the study.

\section{Consent for publication}

Not applicable.

\section{Competing interests}

The authors declare no conflict of interest.

\footnotetext{
Author details

'Programa de Pós-Graduação em Recursos Pesqueiros e Engenharia de Pesca, Universidade Estadual do Oeste do Paraná, Toledo, PR 85903-000, Brazil. ${ }^{2}$ Programa de Pós-graduação em Ciência Animal e Recursos Pesqueiros, Universidade Federal do Amazonas, Manaus, AM 69077-000, Brazil. ${ }^{3}$ Instituto de Ciências Biológicas, Universidade Federal do Amazonas, Manaus, AM 69077-000, Brazil. ${ }^{4}$ Instituto Federal de Educação, Ciência e Tecnologia do Amazonas, Presidente Figueiredo, AM 69735-000, Brazil. ${ }^{5}$ Instituto Federal de Educação, Ciência e Tecnologia do Amazonas, Manaus, AM 69020-120, Brazil.
}

Received: 7 January 2021 Accepted: 9 June 2021

Published online: 29 June 2021

\section{References}

1. Marques JGW. Etnoictiologia: pescando pescadores nas águas da transdiciplinaridade. Revista Ouricuri. 2012;2:9-36.

2. Albuquerque UP, Silva JS, Campos JLA, Sousa RS, Silva TC, Alves RRN. The current status of ethnobiological research in Latin America: gaps and perspectives. J Ethnobiol Ethnomed. 2013:9:2-9.

3. Alves RRN, Silva JS, Chaves LS, Albuquerque UP. Ethnozoology: An overview and current perspectives. In: Alves RRN, Albuquerque UP, editors. Ethnozoology: animals in our lives: United States: Academic Press; 2018. p. $513-21$.

4. Alves RRN, Souto WMS. Ethnozoology in Brazil: current status and perspectives. J Ethnobiol Ethnomed. 2011;7:1-19.

5. Silvano RAM. Pesca artesanal e Etnoictiologia. In: Begossi A, editor. (org.). Ecologia de Pescadores da Mata Atlântica e da Amazônia: Brazil: RIMA; 2013. p. 131-61

6. Begossi A, Salivonchyk S, Lopes PFM, Silvano RAM. Fishers' knowledge on the coast of Brazil. J Ethnobiol Ethnomed. 2016;12(1):2-34. https://doi.org/1 0.1186/s13002-016-0091-1.

7. Silvano RAM, Valbo-Jorgensen J. Beyond fishermen's tales: contribuitions of fishers' local ecological knowledge to fish ecology and fisheries management. Environ Develop Sustainability. 2008;10(5):657-75. https://doi. org/10.1007/s10668-008-9149-0.

8. Araújo JG, Santos MAS, Rebello FK, Isaac VJ. Cadeia comercial de peixes ornamentais do rio Xingu, Pará, Brasil. Bol Inst Pesca. 2017:43(2):297-307. https://doi.org/10.20950/1678-2305.2017v43n2p297.

9. Carvalho Júnior JR, Carvalho NASS, Nunes JLG, Camões A, Bezerra MFC, Santana AR, et al. Sobre a pesca de peixes ornamentais por comunidades do rio Xingu, Pará- Brasil: relato de caso. B Inst Pesca. 2009;35:521-30.

10. Lima EMM, Santos PRB, Braga TMP. McGrath DG. A pesca de acari (Pterygoplichthys pardalis) na várzea do Baixo Amazonas, Pará, Brasil: Aspectos estruturais e socioeconômicos. Gaia Sci. 2019;13:70-85. https://doi. org/10.22478/ufpb.1981-1268.2019v13n4.48781.

11. Souza RL, Mendonça MR. Caracterização da pesca e dos pescadores de peixes ornamentais da região Tefé/AM. Uakari. 2009;5:7-17.

12. Rossoni F, Ferreira E, Zuanon J. Fishery and local ecological knowledge of the discus (Symphysodon aequifasciatus, Pellegrin 1904: Cichlidae) na Reserva de Desenvolvimento Sustentável Piagaçu- Purus, baixo rio Purus, Brasil. B Museu Paraense Emílio Goeldi Ciênc Humanas. 2014;9(1):109-28. https://doi.org/10.1590/S1981-81222014000100008.

13. Anjos HDB, Amorim RMS, Siqueira JÁ, Anjos CR. Exportação de peixes ornamentais do estado do Amazonas, bacia Amazônia, Brasil. Bol Inst Pesca. 2009:35:259-74

14. Duncan WP, Inomata SO, Fernandes MN. Comércio de raias de água doce na região do Médio rio Negro, Estado do Amazonas, Brasil. Rev Bras Engenharia Pesca. 2010;5:13-22.

15. Ferreira VAM, Rodrigues TTE, Yamamoto KC, Freitas CEC, Nogueira AJA. Caracterização socioeconómica da pesca ornamental no município de Barcelos, Amazonas, Brasil. Observatorio Econ Latinoam. 2017:2:1-21.

16. Ferreira VAM, Rodrigues TTE, Silva PG, Freitas CEC, Yamamoto KC. Avaliação do comércio de peixes ornamentais no Estado do Amazonas-Brasil. Observatorio Econ Latinoam. 2020;2:1-21.

17. Oliveira AT, Ladislau DS, Ribeiro MWS, Bassul LA, Paiva AJV, Cardoso LD, et al. Conhecimento tradicional de pescadores de arraias de água doce da região Amazônica. Rev Ibero-Am Ciênc Ambientais. 2020;11(2):129-35. https://doi.org/10.6008/CBPC2179-6858.2020.002.0015.

18. Sobreiro T. Dinâmica socioecológica e resiliência da pesca ornamental em Barcelos, Rio Negro, Amazonas, Brasil. Sustentabilidade em Debate. 2016; 7(2):118-34. https://doi.org/10.18472/SustDeb.v7n2.2016.15127.

19. Ladislau DS, Ribeiro MWS, Castro PDS, Aride PHR, Paiva AJV, Polese MF, et al. Ornamental fishing in the region of Barcelos, Amazonas: socioeconomic description and scenario of activity in the view of "piabeiros". Braz J Biol. 2020;80(3):544-56. https://doi.org/10.1590/1519-6984.215806.

20. Zehev BS, Vera A, Asher B, Raimundo R. Ornamental fishery in Rio Negro (Amazon region), Brazil: combining social, economic and fishery analyses. Fisheries Aquaculture J. 2015;6(04):2-4. https://doi.org/10.4172/2150-3508.1 000143. 
21. Chao NL, Petry P, Prang G. Conservation and management of ornamental fish Resources of the Rio Negro Basin, Amazonia, Brazil - Project Piaba. Manaus: Editora Universidade do Amazonas; 2001. p. 310.

22. Montgomery SY. Amazon adventure: how tiny fish are saving the world's largest rainforest: Editora: United States: HMH Books for Young Readers; 2017. p. 80.

23. Beltrão H, Zuanon J, Ferreira E. Checklist of the ichthyofauna of the Rio Negro basin in the Brazilian Amazon. Zookeys. 2019;881:53-89 https://doi. org/10.3897/zookeys.881.32055.

24. Instituto Brasileiro de Geografia e Estatística-IBGE (Brazil). Censo demográfico 2010: Barcelos, Amazonas. [electronic version: https://cidades. ibge.gov.br/brasil/am/barcelos/panorama]. Accessed 30 Nov 2020

25. Anjos HDB, Yamamoto KC, Magalhães ERS. Biologia reprodutiva e hábitos alimentares do rodóstomo (Hemigrammus bleheri) um peixe ornamental da bacia do Médio Rio Negro, Estado do Amazonas, Brasil. Bol Inst Pesca. 2017; 43:65-77. https://doi.org/10.20950/1678-2305.2017v43n1p65.

26. Begossi A, Clauzet M, Figueiredo JL, Garuana L, Lima RV, Lopes PF, et al. Are biological species and higher-ranking categories real? Fish folk taxonomy on Brazil's Atlantic forest coast and in the Amazon. Curr Anthropol. 2008; 49(2):291-306. https://doi.org/10.1086/527437.

27. Sioli H. Amazônia: fundamentos de ecologia da maior região de florestas tropicais. Vozes Ltda Petrópolis-RJ. 1985;1:15-44.

28. Loebens SC, Farias EU, Yamamoto KC, Freitas CEC. Diversidade de assembleias de peixes em floresta alagada de águas pretas da Amazônia Central. Rev Sci Amazonia. 2016;5:37-44.

29. Loebens SC, Farias EU, Freitas CEC, Yamamoto KC. Influence of hydrological cycle on the composition and structure of fish assemblages in an Igapó Forest, Amazonas, Brazil. Bol Inst Pesca. 2019;45(1):1-10. https://doi.org/10.2 0950/1678-2305.2019.45.1.432.

30. Walker I. The food spectrum of the cardinal-tetra (Paracheirodon axelrodi, Characidae) in its natural habitat. Acta Amazonica. 2004;34(1):69-73. https:// doi.org/10.1590/S0044-59672004000100009.

31. Crampton WGR. Ecology and life history of an Amazon floodplain cichlid: the discus fish Symphysodon (Perciformes: Cichlidae). Neotrop Ichthyol. 2008:4(4):599-612. https://doi.org/10.1590/\$1679-62252008000400008.

32. Duncan WP, Fernandes MN. Physicochemical characterization of the White, black, and Clearwater rivers of the Amazon basin and its implications on the distribution of freshwater stingrays (Chondrichthyes, Potamotrygonidae). Pan-Am J Aquat Sci. 2010;5:454-64.

33. Oliveira AT, Araújo MLG, Lemos JRG, Santos MQC, Pantoja-Lima J, Aride PHR, et al. Ecophysiological interactions and water-related physicochemial parameters among freshwater stingrays. Braz J Biol. 2017;77(3):616-21. https://doi.org/10.1590/1519-6984.01816.

34. Chellappa S, Câmara MR, Verani JR. Ovarian development in the amazonian red discus, Symphysodon discus Heckel (Osteichthyes: Cichlidae). Braz J Biol. 2005;65(4):609-16. https://doi.org/10.1590/S1519-69842005000400007.

35. Zuanon J, Mendonça FP, Santo HMVE, Dias MS, Galuch AV, Akama A. Guia de peixes da Reserva Adolpho Ducke. INPA. 2015;1:155.

36. Shibuya A, Araujo MLG, Zuanon JAS. Analysis of stomach contents of freshwater stingrays (Elasmobranchii, Potamotrygonidae) from the middle Negro River, Amazonas, Brazil. Pan-Am J Aquat Sci. 2009:4:466-75.

37. Shibuya A, Zuanon J, Tanaka S. Feeding behavior of the Neotropical freshwater stingray Potamotrygon motoro (Elasmobranchii: Potamotrygonidae). Neotrop Ichthyol. 2009;10(1):189-96. https://doi.org/10.1 590/S1679-62252012000100018

38. Isaac VJ, Fabré NN, Silva CO, Ruffino ML, Saint-Paul U. Ecologia da fauna ictíica. In: Batista VS, Isaac VJ. (org.). Peixes e pesca no Solimões-Amazonas: uma avaliação integrada. Ibama/ProVárzea. 2012;1:207-49.

\section{Publisher's Note}

Springer Nature remains neutral with regard to jurisdictional claims in published maps and institutional affiliations.

Ready to submit your research? Choose BMC and benefit from:

- fast, convenient online submission

- thorough peer review by experienced researchers in your field

- rapid publication on acceptance

- support for research data, including large and complex data types

- gold Open Access which fosters wider collaboration and increased citations

- maximum visibility for your research: over $100 \mathrm{M}$ website views per year

At BMC, research is always in progress.

Learn more biomedcentral.com/submissions 